6-8-2021

\title{
Extending Singh-Maddala Distribution
}

Mohamed Ali Ahmed

Al Madina Higher Institute of Management and Technology, Giza, Egypt, mrmohamedali2005@yahoo.com

Follow this and additional works at: https://digitalcommons.wayne.edu/jmasm

Part of the Applied Statistics Commons, Social and Behavioral Sciences Commons, and the Statistical Theory Commons

\section{Recommended Citation}

Ahmed, Mohamed Ali (2021) "Extending Singh-Maddala Distribution," Journal of Modern Applied Statistical Methods: Vol. 19 : Iss. 1 , Article 11.

DOI: $10.22237 / \mathrm{jmasm} / 1608553680$

Available at: https://digitalcommons.wayne.edu/jmasm/vol19/iss1/11

This Regular Article is brought to you for free and open access by the Open Access Journals at DigitalCommons@WayneState. It has been accepted for inclusion in Journal of Modern Applied Statistical Methods by an authorized editor of DigitalCommons@WayneState. 


\title{
Extending Singh-Maddala Distribution
}

\author{
Mohammed Ali Ahmed \\ Al Madina Higher Institute of Management and Technology \\ Giza, Egypt
}

\begin{abstract}
A new distribution, the exponentiated transmuted Singh-Maddala distribution (ETSM), is presented, and three important special distributions are illustrated. Some mathematical properties are obtained, and parameters estimation method is applied using maximum likelihood. Illustrations based on random numbers and a real data set are given.
\end{abstract}

Keywords: Singh-Maddala distribution, moments, order statistics, quantile function, maximum likelihood estimation

\section{Introduction}

The Burr distribution was first discussed by Burr (1942) as a two-parameter family; it is a very flexible distribution that can express a wide range of distributions shapes. The Singh-Maddala (SM) distribution introduced by Singh and Maddala (1976). It is known under various other names, such as the Burr XII distribution (Tadikamalla, 1980; Al-Khazaleh, 2016), the Pareto IV (Arnold, 1983) distribution, beta-P (Mielke \& Johnson, 1974) distribution and generalized log-logistic (El-Saidi et al., 1990) distribution. The SM distribution includes, overlaps, or has as a limiting case many commonly-used distributions such as gamma, lognormal, log logistic, bellshaped, and J-shaped beta distributions (but not U-shaped). The SM distribution is used in various fields such as finance, hydrology, and reliability to model a variety of data types.

Generally, the cumulative distribution function (CDF) of the transmuted function (Aryal \& Tsokos, 2011) is given by

$$
\mathrm{F}(x)=(1+\lambda) \mathrm{G}(x)-(\lambda \mathrm{G}(x))^{2} ;|\lambda| \leq 1,-\infty<x<\infty,
$$

then, the CDF of the exponentiated transmuted function is defined by

doi: 10.22237/jmasm/1608553680 | Accepted: October 4, 2018; Published: June 8, 2021.

Correspondence: Mohamed Ali Ahmed, mrmohamedali2005@yahoo.com 


$$
\mathrm{F}(x)=\mathrm{Z}^{v}(x)=\left[(1+\lambda) \mathrm{G}(x)-\lambda(\mathrm{G}(x))^{2}\right]^{v} ;-\infty<x<\infty .
$$

The aim of this study is to present and study a new distribution called the ETSM distribution based on the exponentiated transmuted function.

\section{The CDF and PDF of the ETSM Distribution}

The CDF and the probability density function (PDF) of the Singh and Maddala (Singh \& Maddala, 1976) are, respectively,

$$
\mathrm{G}(x ; a, b, p)=1-\left[1+\left(\frac{x}{b}\right)^{a}\right]^{-p} ; x \geq 0 ; a, b, p>0
$$

and

$$
\mathrm{g}(x ; a, b, p)=\left(\frac{a p}{b}\right)\left(\frac{x}{b}\right)^{a-1}\left[1+\left(\frac{x}{b}\right)^{a}\right]^{-(p+1)}, x \geq 0, a, b, p>0 .
$$

The exponentiated transmuted Singh-Maddala (ETSM) distribution can be derived easily by substituting equation (3) into equation (2); it yields the CDF of the $\operatorname{ESTM}(a, b, p, v, \lambda)$ distribution as follows:

$$
\mathrm{F}(x)=\left\{(1+\lambda)\left[1-\left(1+\left(\frac{x}{b}\right)^{a}\right)^{-p}\right]-\lambda\left[1-\left(1+\left(\frac{x}{b}\right)^{a}\right)^{-p}\right]^{2}\right\}^{v}
$$

where $v, a$, and $p$ are shape parameters and $b$ and $\lambda$ are scale parameters. Differentiating equation (5) yields the PDF of the ETSM distribution: 


\section{EXTENDING SINGH-MADDALA DISTRIBUTION}

$$
\begin{aligned}
f(x)=\left(\frac{v a p}{b}\right)\left(\frac{x}{b}\right)^{a-1}\left(1+\left(\frac{x}{b}\right)^{a}\right)^{-(p+1)} \\
\times\left\{(1+\lambda)\left[1-\left(1+\left(\frac{x}{b}\right)^{a}\right)^{-p}\right]-\lambda\left[1-\left(1+\left(\frac{x}{b}\right)^{a}\right)^{-p}\right]^{2}\right\}^{v-1} \\
\\
\times\left\{(1+\lambda)-2 \lambda\left[1-\left(1+\left(\frac{x}{b}\right)^{a}\right)^{-p}\right]\right\}
\end{aligned}
$$

The ETSM distribution has several special cases as follows: setting $\lambda=0$ gives the exponentiated Singh-Maddala (ESM) distribution, setting $v=1$ gives the transmuted Singh-Maddala (TSM) distribution, and setting $\lambda=0$ and $v=1$ gives the Singh-Maddala (SM) distribution. Displayed in Figure 1 are plots of the ETSM density for some values of the parameters $a, b, p, v$, and $\lambda$.

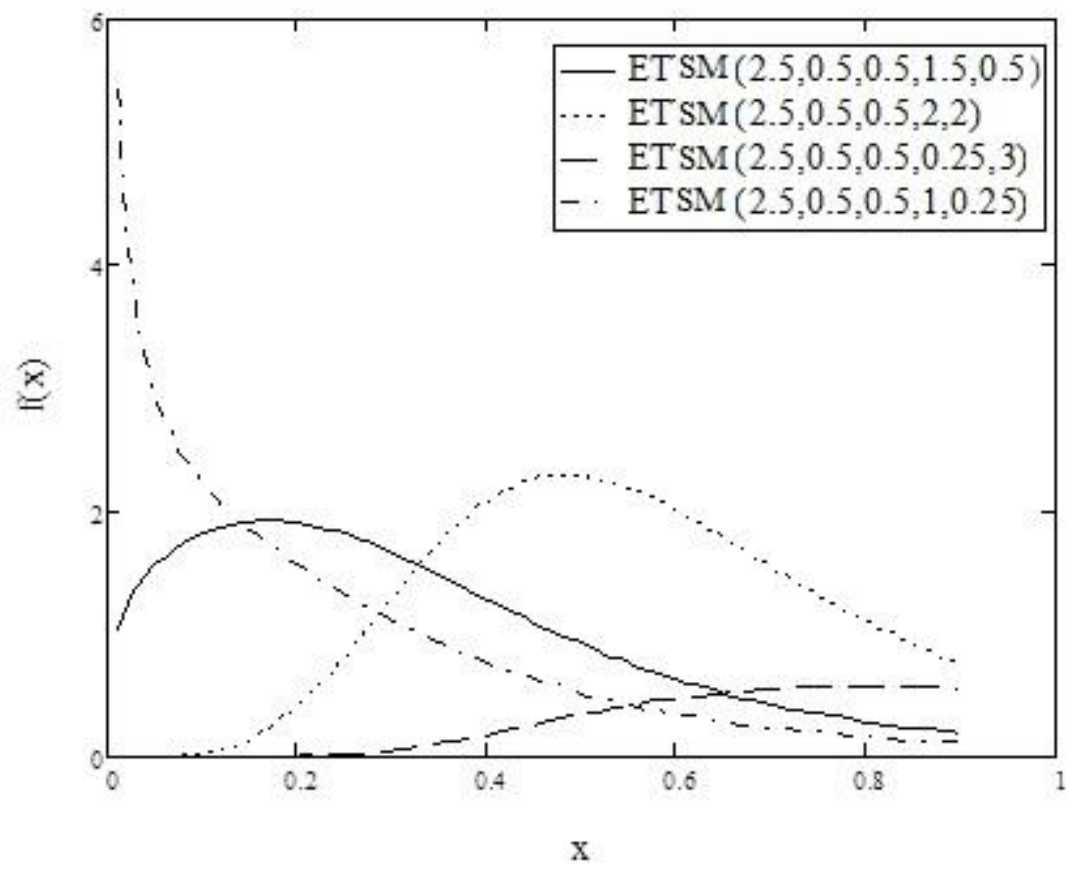

Figure 1. The PDF of the ETSM distribution with different parameters 


\section{Statistical Properties}

\section{The Quantile Function of the ETSM Distribution}

The quantile function of the ETSM distribution is derived in the following Corollary

Corollary 1. The quantile function of the random variable $X$ having the CDF of the ETSM distribution is given by the nonlinear equation

$$
x_{q}=\left[\frac{q^{1 / v}}{\left(\lambda b^{-2 a p}-(1+\lambda) x_{q}^{1 / 2} b^{a p}\right)}\right]^{-\frac{1}{2 a p}} .
$$

Proof. $\quad$ Equating $q$ to the CDF,

$$
q=\mathrm{p}\left(X \leq x_{q}\right)=\mathrm{F}\left(x_{q}\right)=q ; x_{q}>0,0<q<1 .
$$

Then

$$
q^{\frac{1}{v}}=(1+\lambda)\left(1-\left[1+(x)^{a}\right]^{-p}\right)-\lambda\left(1-\left[1+\left(\frac{x}{b}\right)^{a}\right]^{-p}\right)^{2}
$$

and

$$
x_{q}=\left[\frac{q^{1 / v}}{\left(\frac{\lambda}{b^{-2 a p}}-\frac{1+\lambda}{b^{-a p} x_{q}^{1 / 2}}\right)}\right]^{-\frac{1}{2 a p}},
$$

where the last equation is a nonlinear quantile function and it needs a numerical solution to be solved. 


\section{EXTENDING SINGH-MADDALA DISTRIBUTION}

\section{The $r^{\text {th }}$ Moment}

The $r^{\text {th }}$ moment of a random variable $X$ of the ETSM distribution can be obtained from the following theorem:

Theorem 1. The $r^{\text {th }}$ moment of the random variable $X$ having the PDF of the ETSM distribution is given by

$$
\begin{aligned}
& \mathrm{E}\left(X^{r}\right)= \\
& \quad(-1)^{r / a} b^{r}-\left(\frac{r b^{r}}{a p}\right)\left(\sum_{i=0}^{v} \sum_{j=0}^{\infty}\left(\begin{array}{c}
v \\
i
\end{array}\right)\left(\begin{array}{c}
r / a-1 \\
j
\end{array}\right)(-1)^{r / a-1-j}(\lambda)^{i} \mathrm{~B}\left(\frac{i-j+1}{p}, v+1\right)\right)
\end{aligned}
$$

Proof. The $r^{\text {th }}$ moment of a random variable $X$ can be obtained from

$$
\mathrm{E}\left(x^{r}\right)=\int_{x} x^{r} \mathrm{f}(x) d x
$$

Then, substituting equation (6) into (10) yields

$$
\begin{aligned}
& \mathrm{E}\left(X^{r}\right)=v \int_{0}^{\infty} x^{r}\left[(1+\lambda)\left(1-\left[1+\left(\frac{x}{b}\right)^{a}\right]^{-p}\right)-\lambda\left(1-\left[1+\left(\frac{x}{b}\right)^{a}\right]^{-p}\right)^{2}\right]^{\nu-1} \\
& \times\left(\frac{a p}{b}\right)\left(\frac{x}{b}\right)^{a-1}\left[1+\left(\frac{x}{b}\right)^{a}\right]^{-(p+1)}\left[(1+\lambda)-2 \lambda\left(1-\left[1+\left(\frac{x}{b}\right)^{a}\right]^{-p}\right] d x\right.
\end{aligned}
$$

Setting $y=1-\left[1+(x / b)^{a}\right]^{-p}$ gives $x^{r}=b^{r}\left[(1-y)^{-1 / p}-1\right]^{r / a}$. Substituting this into (11) yields

$$
\mathrm{E}\left(Y^{r}\right)=\int_{0}^{1} v b^{r}\left[(1-y)^{-1 / p}-1\right]^{\frac{r}{a}}\left(y+\lambda y-\lambda y^{2}\right)^{v-1}(1+\lambda-2 \lambda y) d y .
$$

Then, integration by parts and binomial expansion yield 


$$
\begin{aligned}
& \mathrm{E}\left(X^{r}\right)= \\
& (-1)^{r / a} b^{r}-\left(\frac{r b^{r}}{a p}\right)\left(\sum_{i=0}^{v} \sum_{j=0}^{\infty}\left(\begin{array}{c}
v \\
i
\end{array}\right)\left(\begin{array}{c}
r / a-1 \\
j
\end{array}\right)(-1)^{r / a-1-j}(\lambda)^{i} \mathrm{~B}\left(\frac{i-j+1}{p}, v+1\right)\right)
\end{aligned}
$$

Setting $r=0$ gives $\mathrm{E}\left(x^{0}\right)=1$. Setting $r=1$ gives

$$
\begin{aligned}
& \mathrm{E}(X)= \\
& (-1)^{1 / a} b-\left(\frac{b}{a p}\right)\left(\sum_{i=0}^{v} \sum_{j=0}^{\infty}\left(\begin{array}{c}
v \\
i
\end{array}\right)\left(\begin{array}{c}
1 / a-1 \\
j
\end{array}\right)(-1)^{1 / a-1-j}(\lambda)^{i} \mathrm{~B}\left(\frac{i-j+1}{p}, v+1\right)\right)
\end{aligned}
$$

Setting $r=2$ gives

$$
\begin{aligned}
& \mathrm{E}\left(X^{2}\right)= \\
& (-1)^{2 / a} b^{2}-\left(\frac{2 b^{2}}{a p}\right)\left(\sum_{i=0}^{v} \sum_{j=0}^{\infty}\left(\begin{array}{l}
v \\
i
\end{array}\right)\left(\begin{array}{c}
2 / a-1 \\
j
\end{array}\right)(-1)^{2 / a-1-j}(\lambda)^{i} \mathrm{~B}\left(\frac{i-j+1}{p}, v+1\right)\right)
\end{aligned}
$$

Similarly, $\mathrm{E}\left(X^{3}\right)$ and $\mathrm{E}\left(X^{4}\right)$ can be calculated. The variance can be given by the fact that $\operatorname{Var}(X)=\mathrm{E}\left(X^{2}\right)-[\mathrm{E}(X)]^{2}$. Therefore, Skewness and Kurtosis can be given, respectively, by

$$
\begin{aligned}
\text { Skewness }(X) & =\frac{\mathrm{E}\left(X^{3}\right)-3 \mathrm{E}(X) \mathrm{E}\left(X^{2}\right)+2 \mathrm{E}^{3}(X)}{\operatorname{Var}^{3 / 2}(X)}, \\
\operatorname{Kurtosis}(X) & =\frac{\mathrm{E}\left(X^{4}\right)-4 \mathrm{E}(X) \mathrm{E}\left(X^{3}\right)+6 \mathrm{E}\left(X^{2}\right) \mathrm{E}^{2}(X)-3 \mathrm{E}^{4}(X)}{\operatorname{Var}^{2}(X)}
\end{aligned}
$$

\section{The Moment Generating Function}

The moment generating function of the ETSM distribution is obtained in the following theorem:

Theorem 2. The moment generating function of the random variable $X$ which has the PDF of the ETSM distribution is given by 


\section{EXTENDING SINGH-MADDALA DISTRIBUTION}

$$
\mathbf{M}_{x}(t)=\sum_{r=0}^{\infty} \frac{(t)^{r}}{r !} \mu_{x}^{r} .
$$

Proof. Clearly, from the following fact

$$
\mathbf{M}_{x}(t)=\mathrm{E}(\exp (x t))
$$

using the expansion of $\exp (x t)$ yields

$$
\mathrm{M}_{x}(t)=\mathrm{E}\left(\sum_{r=0}^{\infty} \frac{(x t)^{r}}{r !}\right)
$$

Then

$$
\mathbf{M}_{x}(t)=\sum_{r=0}^{\infty} \frac{(t)^{r}}{r !} \mathrm{E}\left(x^{r}\right)
$$

and hence

$$
\mathbf{M}_{x}(t)=\sum_{r=0}^{\infty} \frac{(t)^{r}}{r !} \mu_{x}^{r} .
$$

\section{The Mode}

The log function of the PDF is 


\section{MOHAMED ALI AHMED}

$$
\begin{aligned}
\log \mathrm{f}(x) & =\log v+(v-1) \log \left[(1+\lambda)\left(1-\left[1+\left(\frac{x}{b}\right)^{a}\right]^{-p}\right)\right. \\
& \left.-\lambda\left(1-\left[1+\left(\frac{x}{b}\right)^{a}\right]^{-p}\right)^{2}\right]+\log \left(\frac{a p}{b}\right)+(a-1) \log \left(\frac{x}{b}\right) \\
& -(p+1) \log \left[1+\left(\frac{x}{b}\right)^{a}\right]+\log \left[(1+\lambda)-2 \lambda\left(1-\left[1+\left(\frac{x}{b}\right)^{a}\right]^{-p}\right)\right]
\end{aligned}
$$

Then

$$
\begin{aligned}
& \frac{d}{d x} \log \mathrm{f}(x)=(v-1)\left\{\frac{(1+\lambda)\left[1+\frac{x^{a}}{b}\right]^{-(p+1)}\left(\frac{a p}{b}\right)\left(\frac{x}{b}\right)^{a-1}}{\left[(1+\lambda)\left(1-\left[1+\left(\frac{x}{b}\right)^{a}\right]^{-p}\right]-\lambda\left(1-\left[1+\left(\frac{x}{b}\right)^{a}\right]^{-p}\right)^{2}\right]}\right. \\
& \left.-\frac{2 \lambda p\left(1-\left[1+\left(\frac{x}{b}\right)^{a}\right]^{-p}\right)\left[1+\left(\frac{x}{b}\right)^{a}\right]^{-(p+1)}\left(\frac{a}{b}\right)\left(\frac{x}{b}\right)^{a-1}}{\left[(1+\lambda)\left(1-\left[1+\left(\frac{x}{b}\right)^{a}\right]^{-p}\right)-\lambda\left(1-\left[1+\left(\frac{x}{b}\right)^{a}\right]^{-p}\right)^{2}\right]}\right\} \\
& +\frac{(a-1)}{x}-(p+1) \frac{\left(\frac{a}{b}\right)\left(\frac{x}{b}\right)^{a-1}}{\left[1+\left(\frac{x}{b}\right)^{a}\right]}-\frac{2 \lambda p\left[1+\left(\frac{x}{b}\right)^{a}\right]^{-(p+1)}\left(\frac{a}{b}\right)\left(\frac{x}{b}\right)^{a-1}}{\left[(1+\lambda)-2 \lambda\left(1-\left[1+\left(\frac{x}{b}\right)^{a}\right]^{-p}\right]\right]}
\end{aligned}
$$

The mode can be determined by equating the previous equation to zero where it is a nonlinear equation and needs a numerical solution, to be solved with respect 


\section{EXTENDING SINGH-MADDALA DISTRIBUTION}

to $x$ on one condition: that the value of $x$ that satisfies the following equation must be less than zero.

$$
\begin{aligned}
& \frac{d^{2}}{d x^{2}} \log f(x)=(v-1)\left\{\frac{\left[(1+\lambda)\left(1-B_{i}\right)-\lambda A_{i}\right]\left[(1+\lambda)\left(\frac{a^{2} p}{b^{2}}\right) D_{i}^{2(a-1)}(p+1) F_{i}^{(p+2)}\right]}{\left[(1+\lambda)\left(1-B_{i}\right)-\lambda A_{i}\right]^{2}}\right. \\
& +\frac{\left[(1+\lambda)\left(1-B_{i}\right)-\lambda A_{i}\right]\left[(1+\lambda) p(a-1) D_{i}^{(a-2)} E_{i}\right]}{\left[(1+\lambda)\left(1-B_{i}\right)-\lambda A_{i}\right]^{2}} \\
& -\frac{\left[(1+\lambda)\left(1-B_{i}\right)-\lambda A_{i}\right]\left[2 \lambda p F_{i}^{(p+2)}\left(\frac{a^{2}}{b^{2}}\right) D_{i}^{2(a-1)}(p+1)\left(1-B_{i}\right)\right]}{\left[(1+\lambda)\left(1-B_{i}\right)-\lambda A_{i}\right]^{2}} \\
& +\frac{\left[(1+\lambda)\left(1-B_{i}\right)-\lambda A_{i}\right]\left[2 \lambda p\left(\frac{a}{b^{3}}\right)(a-1) D_{i}^{(a-2)}\left(1-B_{i}\right) E_{i}\right]}{\left[(1+\lambda)\left(1-B_{i}\right)-\lambda A_{i}\right]^{2}} \\
& \left.-\frac{\left[(1+\lambda)\left(\frac{a p}{b}\right) D_{i}^{(a-1)} E_{i}-2 \lambda\left(\frac{a p}{b}\right) D_{i}^{(a-1)} E_{i}\left(1-B_{i}\right)\right]}{\left[(1+\lambda)\left(1-B_{i}\right)-\lambda A_{i}\right]^{2}}\right\} \\
& -\frac{(a-1)}{x^{2}}-(p+1) \frac{\left(1+C_{i}\right)(a-1)\left(\frac{a}{b^{3}}\right) D_{i}^{(a-2)}-\left(\frac{a}{b^{3}}\right) D_{i}^{(a-1)}}{\left(1+C_{i}\right)^{2}} \\
& +\frac{\left((1+\lambda)-2 \lambda\left(1-B_{i}\right)\right)\left(2 \lambda p(p+1)\left(\frac{a^{2}}{b^{2}}\right) D_{i}^{2(a-1)} F_{i}^{(p+2)}-2 \lambda p(a-1)\left(\frac{a}{b^{3}}\right) D_{i}^{(a-2)} E_{i}\right)}{\left((1+\lambda)-2 \lambda\left(1-B_{i}\right)\right)^{2}} \\
& -\frac{\left(-\left(\frac{2 \lambda p a}{b}\right) D_{i}^{(a-1)} E_{i}\right)^{2}}{\left((1+\lambda)-2 \lambda\left(1-B_{i}\right)\right)^{2}}
\end{aligned}
$$




\section{MOHAMED ALI AHMED}

\section{Reliability Properties}

Properties of reliability (Meeker \& Escobar, 1998) will be obtained.

\section{The Survival Function}

Because

$$
\overline{\mathrm{F}}(x)=1-\mathrm{F}(x)
$$

the survival function is

$$
\overline{\mathrm{F}}(x)=1-\left[(1+\lambda)\left(1-\left[1+\left(\frac{x}{b}\right)^{a}\right]^{-p}\right)-\lambda\left(1-\left[1+\left(\frac{x}{b}\right)^{a}\right]^{-p}\right)^{2}\right]^{\nu}
$$

\section{The Hazard Rate Function}

The hazard rate function of the ETSM distribution is derived in the following Corollary:

Corollary 2. The hazard function of the random variable $X$ having CDF and PDF of the ETSM Distribution is given by

$$
\begin{aligned}
& \mathrm{h}(x)= \frac{v\left[(1+\lambda)\left(1-\left[1+\left(\frac{x}{b}\right)^{a}\right]^{-p}\right)-\lambda\left(1-\left[1+\left(\frac{x}{b}\right)^{a}\right]^{-p}\right)^{2}\right]^{\nu-1}}{1-\left[(1+\lambda)\left(1-\left[1+\left(\frac{x}{b}\right)^{a}\right]^{-p}\right)-\lambda\left(1-\left[1+\left(\frac{x}{b}\right)^{a}\right]^{-p}\right)^{2}\right]^{\nu}} \\
& \times\left(\frac{a p}{b}\right)\left(\frac{x}{b}\right)^{a-1}\left[1+\left(\frac{x}{b}\right)^{a}\right]^{-(p+1)}\left[(1+\lambda)-2 \lambda\left(1-\left[1+\left(\frac{x}{b}\right)^{a}\right]^{-p}\right)\right]
\end{aligned}
$$

Proof. Generally, the hazard function of the random variable $X$ is given by 


\section{EXTENDING SINGH-MADDALA DISTRIBUTION}

$$
\mathrm{h}(x)=\frac{\mathrm{f}(x)}{1-\mathrm{F}(x)}
$$

Substituting equations (5) and (6) into the previous equation yields

$$
\begin{aligned}
& \mathrm{h}(x)= \frac{v\left[(1+\lambda)\left(1-\left[1+\left(\frac{x}{b}\right)^{a}\right]^{-p}\right)-\lambda\left(1-\left[1+\left(\frac{x}{b}\right)^{a}\right]^{-p}\right)^{2}\right]^{\nu-1}}{1-\left[(1+\lambda)\left(1-\left[1+\left(\frac{x}{b}\right)^{a}\right]^{-p}\right)-\lambda\left(1-\left[1+\left(\frac{x}{b}\right)^{a}\right]^{-p}\right)^{2}\right]^{\nu}} \\
& \times\left(\frac{a p}{b}\right)\left(\frac{x}{b}\right)^{a-1}\left[1+\left(\frac{x}{b}\right)^{a}\right]^{-(p+1)}\left[(1+\lambda)-2 \lambda\left(1-\left[1+\left(\frac{x}{b}\right)^{a}\right]^{-p}\right)\right]
\end{aligned}
$$

Figure 2 illustrates the Hazard function of the ETSM distribution with different parameters. One can see, in Figure 2, two types of Hazard functions curves of the ETSM distribution are described as follows: An increasing then constant then decreasing Hazard curve and an increasing then decreasing Hazard curve.

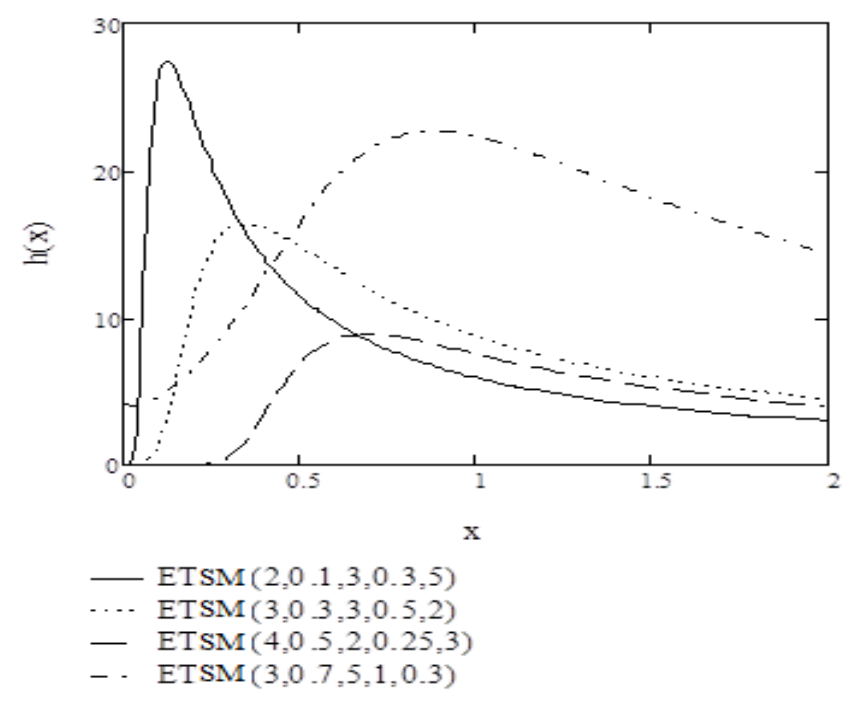

Figure 2. The hazard function of the ESTM distribution with different parameters 


\section{The Cumulative Hazard Rate Function}

Based on

$$
\mathrm{H}(x)=\int_{0}^{x} \mathrm{~h}(x) d x
$$

and substituting equation (8) into the previous equation yields

$$
\begin{aligned}
\mathrm{H}(x) & =\int_{0}^{x} \mathrm{~h}(x) d x \\
& =-\ln \left[1-\lambda\left(\frac{x}{b}\right)^{-2 a p}-(1+\lambda)\left(\frac{x}{b}\right)^{-a p}\right]^{v}
\end{aligned}
$$

\section{Order Statistics of the ESTM Distribution}

The $r^{\text {th }}$ moment of order statistics of the ETSM distribution (Arnold et al., 1992) is derived in the following theorem:

Theorem 3. The density $\mathrm{f}_{u: n}\left(x_{u}\right)$ of the $u^{\text {th }}$ order statistic, for $u=1,2, \ldots, n$, from iid random variables $X_{1}, X_{2}, \ldots, X_{n}$ following the ETSM distribution (Arnold et al., 1992) is given by

$$
\begin{aligned}
\mathrm{f}_{u: n}\left(x_{u}\right)= & \frac{1}{\beta(u, n-u+1)} \\
\times & \times \sum_{w=0}^{n-u}(-1)^{w}\left(\begin{array}{c}
n-u \\
w
\end{array}\right) v\left[(1+\lambda)\left(1-\left[1+\left(\frac{x}{b}\right)^{a}\right]^{-p}\right)\right. \\
& \left.-\lambda\left(1-\left[1+\left(\frac{x}{b}\right)^{a}\right]^{-p}\right)^{2}\right]^{v-1+v u+v w-v} \\
& \times\left(\frac{a p}{b}\right)\left(\frac{x}{b}\right)^{a-1}\left[1+\left(\frac{x}{b}\right)^{a}\right]^{-(p+1)}\left[(1+\lambda)-2 \lambda\left(1-\left[1+\left(\frac{x}{b}\right)^{a}\right]^{-p}\right)\right]
\end{aligned}
$$




\section{EXTENDING SINGH-MADDALA DISTRIBUTION}

Proof. Generally, the density $\mathrm{f}_{u: n}\left(x_{u}\right)$ of the $u^{\text {th }}$ order statistic, for $u=1,2, \ldots$, $n$, from iid random variables $X_{1}, X_{2}, \ldots, X_{n}$ (Arnold et al., 1992) is given by

$$
\mathrm{f}_{u: n}\left(x_{u}\right)=\frac{\mathrm{f}\left(x_{u}\right)}{\beta(u, n-u+1)} \mathrm{F}\left(x_{u}\right)^{u-1}\left\{1-\mathrm{F}\left(x_{u}\right)\right\}^{n-u}
$$

Using binomial expansion yields

$$
\mathrm{f}_{u: n}\left(x_{u}\right)=\frac{\mathrm{f}\left(x_{u}\right)}{\beta(u, n-u+1)} \sum_{w=0}^{n-u}(-1)^{w}\left(\begin{array}{c}
n-u \\
w
\end{array}\right) \mathrm{F}\left(x_{u}\right)^{u+w-1}
$$

Then,

$$
\begin{aligned}
f_{u: n}\left(x_{u}\right)= & \frac{1}{\beta(u, n-u+1)} \\
\times & \times \sum_{w=0}^{n-u}(-1)^{w}\left(\begin{array}{c}
n-u \\
w
\end{array}\right) v\left[(1+\lambda)\left(1-\left[1+\left(\frac{x}{b}\right)^{a}\right]^{-p}\right)\right. \\
& \left.-\lambda\left(1-\left[1+\left(\frac{x}{b}\right)^{a}\right]^{-p}\right)^{2}\right]^{v-1+v u+v w-v} \\
& \times\left(\frac{a p}{b}\right)\left(\frac{x}{b}\right)^{a-1}\left[1+\left(\frac{x}{b}\right)^{a}\right]^{-(p+1)}\left[(1+\lambda)-2 \lambda\left(1-\left[1+\left(\frac{x}{b}\right)^{a}\right]^{-p}\right)\right]
\end{aligned}
$$

Moments of order statistics can be given by

$$
\mathrm{E}\left(X_{u: n}^{r}\right)=\int_{0}^{\infty} x_{u}^{r} \mathrm{f}_{u: n}\left(x_{u}\right) d x_{u}
$$

Substituting equation (6) into the previous equation yields 


$$
\begin{aligned}
& \mathrm{f}_{u: n}\left(x_{u}\right)=\frac{1}{\beta(u, n-u+1)} \\
& \times \sum_{w=0}^{n-u}(-1)^{w}\left(\begin{array}{c}
n-u \\
w
\end{array}\right) v \int_{0}^{\infty} x_{u}^{r}\left[(1+\lambda)\left(1-\left[1+\left(\frac{x}{b}\right)^{a}\right]^{-p}\right)\right. \\
& \left.\quad-\lambda\left(1-\left[1+\left(\frac{x}{b}\right)^{a}\right]^{-p}\right)^{2}\right]^{v-1+v u+v w-v} \\
& \times\left(\frac{a p}{b}\right)\left(\frac{x}{b}\right)^{a-1}\left[1+\left(\frac{x}{b}\right)^{a}\right]^{-(p+1)}\left[(1+\lambda)-2 \lambda\left(1-\left[1+\left(\frac{x}{b}\right)^{a}\right]^{-p}\right)\right] d x_{u}
\end{aligned}
$$

Setting $y=1-\left[1+(x / b)^{a}\right]^{-p}$ gives $x^{r}=b^{r}\left[(1-y)^{-1 / p}-1\right]^{r / a}$. Substituting this into (23) yields

$$
\mathrm{E}\left(y^{r}\right)=\int v b^{r}\left[(1-y)^{(-1 / p)}-1\right]^{(r / a)}\left(y+\lambda y-\lambda y^{2}\right)^{v u+v w}(1+\lambda-2 \lambda y) d y .
$$

Using integration by parts and binomial expansion yields

$$
\begin{aligned}
\mathrm{E}\left(X^{r}\right) & =\frac{v}{v u+v w}(-1)^{r / a} b^{r}-\frac{v}{v u+v w}\left(\frac{r b^{r}}{a p}\right) \\
& \times \sum_{i=0}^{\infty} \sum_{j=0}^{\infty}\left(\begin{array}{c}
v u+v w \\
i
\end{array}\right)\left(\begin{array}{c}
r / a-1 \\
j
\end{array}\right)(-1)^{r / a-1-j}(\lambda)^{i} \mathrm{~B}\left(\frac{i-j+1}{p}, v u+v w+1\right)
\end{aligned}
$$

\section{Maximum Likelihood Estimation}

Let $X_{1}, X_{2}, \ldots, X_{n}$ be iid random variables following the $\operatorname{ETSM}(a, b, p, \lambda, v)$ distribution; then the likelihood function (Garthwait et al., 1995) is given by 


\section{EXTENDING SINGH-MADDALA DISTRIBUTION}

$$
\begin{aligned}
L=v^{n} \prod_{i=1}^{n}[ & \left.(1+\lambda)\left(1-\left[1+\left(\frac{x_{i}}{b}\right)^{a}\right]^{-p}\right)-\lambda\left(1-\left[1+\left(\frac{x_{i}}{b}\right)^{a}\right]^{-p}\right)^{2}\right]^{\nu-1} \\
& \times\left(\frac{a p}{b}\right)\left(\frac{x_{i}}{b}\right)^{a-1}\left[1+\left(\frac{x_{i}}{b}\right)^{a}\right]^{-(p+1)}\left[(1+\lambda)-2 \lambda\left(1-\left[1+\left(\frac{x_{i}}{b}\right)^{a}\right]^{-p}\right)\right]
\end{aligned}
$$

Hence, the log likelihood function is

$$
\begin{aligned}
\ell(x i ; B)=n \log v & +\log \prod_{i=1}^{n}\left[(1+\lambda)\left(1-\left[1+\left(\frac{x_{i}}{b}\right)^{a}\right]^{-p}\right)-\lambda\left(1-\left[1+\left(\frac{x_{i}}{b}\right)^{a}\right]^{-p}\right)^{2}\right]^{v-1} \\
& +\log \prod_{i=1}^{n}(a p / b)\left(x_{i} / \mathrm{b}\right)^{a-1}\left[1+\left(x_{i} / \mathrm{b}\right)^{a}\right]^{-(p+1)} \\
& +\log \prod_{i=1}^{n}\left[(1+\lambda)-2 \lambda\left(1-\left[1+\left(\frac{x_{i}}{b}\right)^{a}\right]^{-p}\right)\right]
\end{aligned}
$$

Then

$$
\begin{aligned}
\ell(x i ; B)= & n \log v+(v-1) \log \prod_{i=1}^{n}\left[(1+\lambda)\left(1-\left[1+\left(\frac{x_{i}}{b}\right)^{a}\right]^{-p}\right)\right] \\
& -\prod_{i=1}^{n}\left[\lambda\left(1-\left[1+\left(\frac{x_{i}}{b}\right)^{a}\right]^{-p}\right]^{2}\right]+\log \prod_{i=1}^{n}\left(\frac{a p}{b}\right)\left(\frac{x_{i}}{b}\right)^{a-1}\left[1+\left(\frac{x_{i}}{b}\right)^{a}\right]^{-(p+1)} \\
& +\log \prod_{i=1}^{n}\left[(1+\lambda)-2 \lambda\left(1-\left[1+\left(\frac{x_{i}}{b}\right)^{a}\right]^{-p}\right)\right]
\end{aligned}
$$

Let 


\section{MOHAMED ALI AHMED}

$$
\begin{aligned}
& A_{i}=\left(1-\left[1+\left(\frac{x_{i}}{b}\right)^{a}\right]^{-p}\right)^{2}, B_{i}=\left[1+\left(\frac{x_{i}}{b}\right)^{a}\right]^{-p}, C_{i}=\left(\frac{x_{i}}{b}\right)^{a}, D=\frac{x_{i}}{b}, \\
& E_{i}=\left[1+\left(\frac{x_{i}}{b}\right)^{a}\right]^{-(p+1)}, F_{i}=\left[1+\left(\frac{x_{i}}{b}\right)^{a}\right]^{-1}, \text { and } G_{i}=\left[1+\left(\frac{x_{i}}{b}\right)^{a}\right]
\end{aligned}
$$

Then, differentiating with respect to $a$ yields

$$
\begin{aligned}
\frac{\partial \ell(x ; B)}{\partial a}=(v-1) & \sum_{i=1}^{n} \frac{\left(p E_{i} C_{i}\left(\ln D_{i}\right)\right)\left[(1+\lambda)-2 \lambda\left(1-B_{i}\right)\right]}{\left[(1+\lambda)\left(1-B_{i}\right)-\lambda A_{i}\right]} \\
& +\sum_{i=1}^{n} \frac{\left(\left(\frac{a p}{b}\right) D_{i}^{a-1}\left(\ln D_{i}\right) E_{i}\right)\left[1-(p+1) F_{i} C_{i}\right]}{\left[\left(\frac{a p}{b}\right) D_{i}^{a-1} E_{i}\right]} \\
& +\sum_{i=1}^{n} \frac{\left[(-2 p \lambda) E_{i} C_{i}\left(\ln D_{i}\right)\right]}{\left[(1+\lambda)-2 \lambda\left(1-B_{i}\right)\right]}
\end{aligned}
$$

differentiating with respect to $b$ yields

$$
\begin{aligned}
\frac{\partial \ell(x ; B)}{\partial b}=(v-1) \sum_{i=1}^{n} \frac{\left(E_{i}\left(\frac{a p}{b}\right) C_{i}\right)\left[-(1+\lambda)+2 \lambda\left(1-B_{i}\right)\right]}{\left[(1+\lambda)\left(1-B_{i}\right)-\lambda A_{i}\right]} \\
-\sum_{i=1}^{n} \frac{\left(\frac{a p}{b^{2}}\right) D_{i}^{a-1} E_{i}\left[-1+(1-a)+a C_{i}(p+1) F_{i}\right]}{\left[\left(\frac{a p}{b}\right) D_{i}^{a-1} E_{i}\right]} \\
+\sum_{i=1}^{n} \frac{\left[(2 p \lambda) E_{i} C_{i}\left(\frac{a}{b}\right)\right]}{\left[(1+\lambda)-2 \lambda\left(1-B_{i}\right)\right]}
\end{aligned}
$$

differentiating with respect to $p$ yields 


\section{EXTENDING SINGH-MADDALA DISTRIBUTION}

$$
\begin{aligned}
\frac{\partial \ell(x ; B)}{\partial p}=(v-1) & \sum_{i=1}^{n} \frac{\left(B_{i} \ln G_{i}\right)\left[\left((1+\lambda)\left(\frac{a p}{b}\right)\right)+2 \lambda\left(1-B_{i}\right)\right]}{\left[(1+\lambda)\left(1-B_{i}\right)-\lambda A_{i}\right]} \\
+ & \sum_{i=1}^{n} \frac{\left(\left(\frac{a}{b}\right) D_{i}^{a-1} E_{i}\right)\left[1+p \ln G_{i}\right]}{\left[\left(\frac{a p}{b}\right) D_{i}^{a-1} E_{i}\right]}-\sum_{i=1}^{n} \frac{\left[(2 \lambda)\left(1-B_{i}\right) \ln G_{i}\right]}{\left[(1+\lambda)-2 \lambda\left(1-B_{i}\right)\right]}
\end{aligned}
$$

differentiating with respect to $v$ yields

$$
\frac{\partial \ell(x ; B)}{\partial v}=\left(\frac{n}{v}\right)+\sum_{i=1}^{n} \log \left[(1+\lambda)\left(1-B_{i}\right)-\lambda A_{i}\right],
$$

and differentiating with respect to $\lambda$ yields

$$
\frac{\partial \ell(x ; B)}{\partial \lambda}=(v-1) \sum_{i=1}^{n} \frac{\left(1-B_{i}\right)-A_{i}}{\left[(1+\lambda)\left(1-B_{i}\right)-\lambda A_{i}\right]}+\sum_{i=1}^{n} \frac{\left[1-2\left(1-B_{i}\right)\right]}{\left[(1+\lambda)-2 \lambda\left(1-B_{i}\right)\right]} .
$$

Let $\boldsymbol{\theta}$ be the vector of the unknown parameters $(a, b, p, \lambda, v)$; then elements of the $5 \times 5$ information matrix $\mathbf{I}(a, b, p, \lambda, v)$ can be obtained by

$$
\mathbf{I}_{i j}(\hat{\boldsymbol{\theta}})=\mathrm{E}\left[-\left.\frac{\partial^{2} \ell(\boldsymbol{\theta})}{\partial \boldsymbol{\theta}_{i} \partial \boldsymbol{\theta}_{j}}\right|_{\boldsymbol{\theta}=\hat{\boldsymbol{\theta}}}\right] .
$$

Then $\mathbf{I}^{-1}(a, b, p, \lambda, v)$ is the variance covariance matrix of the unknown parameters $(a, b, p, \lambda, v)$ and the asymptotic distributions of the maximum likelihood estimators (MLE) parameters are

$$
\sqrt{n}\left(\hat{\boldsymbol{\theta}}_{i}-\boldsymbol{\theta}_{i}\right) \approx N_{5}\left(0, \mathbf{I}^{-1}\left(\hat{\boldsymbol{\theta}}_{i}\right)\right), \quad i=1, \ldots, 5 .
$$

The approximation of the $100(1-\alpha) \%$ confidence intervals for the unknown parameters based on the asymptotic distribution of the $\operatorname{ETSM}(a, b, p, \lambda, v)$ are determined as 


$$
\hat{\boldsymbol{\theta}}_{i} \pm z_{\alpha / 2} \sqrt{\mathbf{I}^{-1}\left(\hat{\boldsymbol{\theta}}_{i}\right)}, \quad i=1, \ldots, 5
$$

where $z_{\alpha / 2}$ is the upper $(\alpha / 2)^{\text {th }}$ percentile of a standard normal distribution.

\section{Illustration}

This purpose is to obtain MLEs of the ETSM distribution parameters using random numbers to study the MLEs sample behavior. Obtaining parameters estimates is described as follows:

Step (1): Generating a random sample $x_{1}, x_{2}, \ldots, x_{n}$ of sizes $n=10,30,50$, and 100 using the ETSM distribution.

Step (2): Selecting parameters values: $a=0.7, b=2, p=2, \lambda=0.3, v=1.5$

Step (3): Solving (26) to (30) by iteration to get MLEs, biases, root of mean squared error (RMSE), and the Pearson type of parameter estimators (Pearson, 1895) of the ETSM distribution.

Step (4): Repeating steps from 1 to 3 10,000 times.

In this study, random numbers samples are generated with Mathcad using conjugate gradient iteration method. All results are illustrated in Table 1.

The more sample size increases the more biases and RMSE decrease. In addition, the sampling distribution of $a$ is a Pearson Type I distribution at all times, the sampling distribution of $p$ is a Pearson Type IV distribution at all times, the sampling distribution of $\lambda$ is a Pearson Type I distribution at all times, the sampling distribution of $v$ is a Pearson Type I distribution at all times, and the sampling distribution of $b$ differs according to sample size. The estimators can be consistent, specially, when sample size increases.

Table 1. Biases and RMSE of parameters estimation within small, medium, and large samples

\begin{tabular}{|c|c|c|c|c|c|c|c|c|}
\hline \multirow{2}{*}{$\begin{array}{r}\text { Sample } \\
\text { Size } \\
\end{array}$} & \multirow[b]{2}{*}{ Parameter } & \multirow{2}{*}{$\begin{array}{r}\text { Mean } \\
\text { for } 1000 \\
\text { times } \\
\end{array}$} & \multicolumn{2}{|c|}{ Biases } & \multicolumn{2}{|c|}{ RMSE } & \multirow{2}{*}{$\begin{array}{r}\text { Pearson } \\
\text { system } \\
\text { coefficient }\end{array}$} & \multirow{2}{*}{$\begin{array}{r}\begin{array}{r}\text { Pearson } \\
\text { type }\end{array} \\
\end{array}$} \\
\hline & & & Each & Total & Each & Total & & \\
\hline \multirow[t]{5}{*}{10} & $a=0.7$ & 1.796 & 1.096 & 4.444 & 1.982 & 9.460 & -0.5520 & I \\
\hline & $b=2$ & 5.436 & 3.436 & & 7.469 & & 0.2650 & IV \\
\hline & $p=2$ & 4.126 & 2.126 & & 4.125 & & 0.2370 & IV \\
\hline & $\lambda=0.3$ & 0.151 & -0.149 & & 0.425 & & -0.2420 & I \\
\hline & $v=1.5$ & 2.986 & 1.486 & & 3.547 & & -0.4150 & I \\
\hline
\end{tabular}




\section{EXTENDING SINGH-MADDALA DISTRIBUTION}

Table 1 (continuous).

\begin{tabular}{|c|c|c|c|c|c|c|c|c|}
\hline \multirow{2}{*}{$\begin{array}{r}\text { Sample } \\
\text { Size }\end{array}$} & \multirow[b]{2}{*}{ Parameter } & \multirow{2}{*}{$\begin{array}{r}\text { Mean } \\
\text { for } 1000 \\
\text { times }\end{array}$} & \multicolumn{2}{|c|}{ Biases } & \multicolumn{2}{|c|}{ RMSE } & \multirow{2}{*}{$\begin{array}{r}\text { Pearson } \\
\text { system } \\
\text { coefficient }\end{array}$} & \multirow{2}{*}{$\begin{array}{r}\text { Pearson } \\
\text { type }\end{array}$} \\
\hline & & & Each & Total & Each & Total & & \\
\hline \multirow[t]{5}{*}{30} & $a=0.7$ & 1.098 & 0.398 & 3.507 & 1.043 & 7.523 & -0.5290 & I \\
\hline & $b=2$ & 5.071 & 3.071 & & 6.331 & & 0.2960 & IV \\
\hline & $p=2$ & 3.207 & 1.207 & & 2.719 & & 0.1980 & IV \\
\hline & $\lambda=0.3$ & 0.237 & -0.063 & & 0.262 & & -0.1510 & I \\
\hline & $v=1.5$ & 2.620 & 1.120 & & 2.823 & & -0.8460 & I \\
\hline \multirow[t]{5}{*}{50} & $a=0.7$ & 0.890 & 0.190 & 2.771 & 0.678 & 5.787 & -5.2560 & I \\
\hline & $b=2$ & 1.796 & 2.392 & & 4.678 & & -0.6430 & I \\
\hline & $p=2$ & 5.436 & 0.965 & & 2.160 & & 0.0098 & IV \\
\hline & $\lambda=0.3$ & 4.126 & -0.049 & & 0.231 & & -0.1360 & I \\
\hline & $v=1.5$ & 0.151 & 0.995 & & 2.537 & & -0.4810 & I \\
\hline \multirow[t]{5}{*}{100} & $a=0.7$ & 0.829 & 0.129 & 2.108 & 0.510 & 4.865 & -3.4980 & I \\
\hline & $b=2$ & 3.865 & 1.865 & & 4.039 & & -0.3960 & I \\
\hline & $p=2$ & 2.704 & 0.704 & & 1.841 & & 0.0022 & IV \\
\hline & $\lambda=0.3$ & 0.255 & -0.045 & & 0.212 & & -0.1150 & I \\
\hline & $v=1.5$ & 2.173 & 0.673 & & 1.914 & & -0.2980 & I \\
\hline \multirow[t]{5}{*}{300} & $a=0.7$ & 0.725 & 0.025 & 0.799 & 0.201 & 1.580 & -3.0129 & I \\
\hline & $b=2$ & 2.730 & 0.730 & & 1.374 & & -0.3210 & I \\
\hline & $p=2$ & 2.301 & 0.301 & & 0.594 & & 0.0010 & IV \\
\hline & $\lambda=0.3$ & 0.281 & -0.019 & & 0.104 & & -0.1010 & I \\
\hline & $v=1.5$ & 1.620 & 0.120 & & 0.455 & & -0.2130 & I \\
\hline
\end{tabular}

\section{Application}

A practical example using a real data set is given to see how the empirical model works. In our example, the different distributions used are the ETSM, ESM, TSM, and SM distributions. The following data represents the lifetime (hours) of candle lamps for 50 devices (https://www.npl.co.uk/)

$$
\begin{aligned}
& 0.172,0.1730 .270,0.200,0.260,0.186,0.186,0.191,0.192,0.196 \\
& 0.202,0.212,0.216,0.217,0.218,0.219,0.224,0.226,0.227,0.227, \\
& 0.233,0.234,0.241,0.244,0.244,0.245,0.247,0.250,0.250,0.252, \\
& 0.253,0.234,0.256,0.235,0.265,0.265,0.265,0.269,0.275,0.276, \\
& 0.278,0.285,0.288,0.290,0.294,0.216,0.234,0.217,0.238,0.204
\end{aligned}
$$




\section{MOHAMED ALI AHMED}

The results of some goodness-of-fit measures and likelihood ratio tests are computed using Mathcad (version 15) and are included in Table 2 and Table 3, respectively. Figure 3 illustrates probability density functions for different distributions which fit the data.

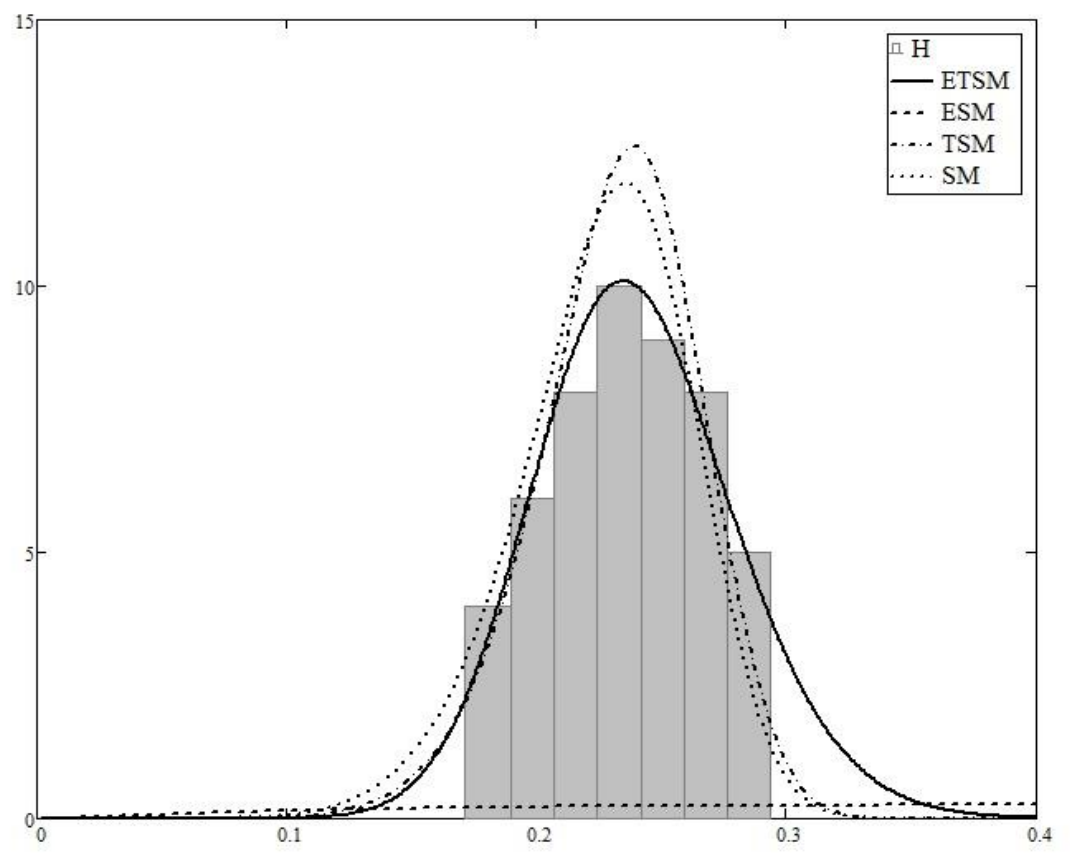

Figure 3. Estimated probability density functions for different distributions

Table 2. The MLE of the parameter(s) and the associated AIC and BIC values

\begin{tabular}{crrrrrrrrrrr} 
& \multicolumn{1}{c}{ MLE of parameters } & & & & & & \\
Model & $\boldsymbol{a}$ & $\boldsymbol{b}$ & $\boldsymbol{p}$ & $\boldsymbol{\lambda}$ & $\boldsymbol{v}$ & KS & $\boldsymbol{p}$-value & AIC & CAIC & BIC \\
\hline ETSM & 6.995 & 0.365 & 19.953 & 0.218 & 1.698 & 0.073 & 0.951 & -189.188 & -187.824 & -179.628 \\
& $(0.107)$ & $(0.024)$ & $(0.171)$ & $(0.056)$ & $(1.331)$ & & & & & \\
ESM & 6.625 & 0.360 & 18.121 & 0.000 & 1.746 & 0.932 & 0.012 & -114.744 & -113.855 & -107.096 \\
& $(2.157)$ & $(0.029)$ & $(6.339)$ & - & $(1.283)$ & & & & & \\
TSM & 9.368 & 0.334 & 13.057 & 0.336 & 1.000 & 0.850 & 0.034 & -123.690 & -123.168 & -117.954 \\
& $(0.632)$ & $(0.055)$ & $(1.275)$ & $(0.068)$ & - & & & & & \\
SM & 8.882 & 0.335 & 14.526 & 0.000 & 1.000 & 0.128 & 0.028 & -183.330 & -182.441 & -175.682 \\
& $(0.118)$ & $(0.054)$ & $(2.744)$ & - & - & & & & & \\
\hline
\end{tabular}




\section{EXTENDING SINGH-MADDALA DISTRIBUTION}

Table 3. The log-likelihood function, likelihood ratio tests statistic, and $p$-values

\begin{tabular}{|c|c|c|c|c|c|}
\hline Model & $\mathrm{H}_{0}$ & $\begin{array}{r}\ell \\
\text { (log likelihood) }\end{array}$ & $\begin{array}{r}\wedge \\
\text { (likelihood ratio } \\
\text { test statistic) }\end{array}$ & $\begin{array}{r}\text { df } \\
\text { (degrees of } \\
\text { freedom) }\end{array}$ & $p$-value \\
\hline ESM & $\lambda=0$ & 95.665 & 7.858 & 1 & $5.06 \mathrm{E}-03$ \\
\hline TSM & $v=0$ & 61.372 & 76.444 & 1 & 0.00 \\
\hline SM & $v=0, \lambda=0$ & 64.845 & 69.498 & 2 & 0.00 \\
\hline
\end{tabular}

Note: The log likelihood of the ETSM $=99.594$

In Table 2, the MLEs of distributions parameters, the corresponding RMSE (given in parentheses), Kolmogorov-Smirnov (KS) test statistic, AIC (Akaike Information Criterion), CAIC (consistent Akaike Information Criterion), and BIC (Bayesian information criterion) are computed for every distribution. The null hypothesis that the data follows the ETSM distribution, only, can be accepted at significance level $\alpha=0.05$ and it is clear that the ETSM distribution has the smallest $\mathrm{KS}$, AIC, CAIC, and BIC, so ETSM distribution can be the best fitted distribution to the data compared with other distributions.

In Table 3, based on the likelihood ratio test, the null hypothesis is that the data follow the nested model and the alternative is the data follow the full model, where the ESM, TSM, and SM distributions are nested by the ETSM distribution. Obviously (from the $p$-values) all null hypotheses can be rejected at the level of significance $\alpha=0.05$, so ETSM distribution can fit the data better than the nested distributions as was illustrated before.

\section{Conclusion}

The ETSM distribution is a useful distribution having flexible statistical properties, wide applications, and generalizes some important distributions. The ETSM distribution can be used quite effectively to provide better fits compared to other distributions.

\section{Acknowledgements}

The authors thank anyone suggested improved comments and the anonymous referees who provided helpful suggestions for this manuscript. 


\section{MOHAMED ALI AHMED}

\section{References}

Al-Khazaleh, A. M. H. (2016). Transmuted Burr type XII distribution: A generalization of the Burr type XII distribution. International Mathematical Forum, 11(12), 547-556. doi: 10.12988/imf.2016.6443

Arnold, C. B. (1983). Pareto distributions. Fairland, MD: International Cooperative Publishing House.

Arnold, C. B., Balakrishnan, N., \& Nagaraja, H. N. (1992). A first course in order statistics. New York: John Wiley and Sons, Inc.

Aryal, G. R., \& Tsokos, C. P. (2011). Transmuted Weibull distribution: A generalization of the Weibull probability distribution. European Journal of Pure and Applied Mathematics, 4(2), 89-102. Retrieved from https://ejpam.com/index.php/ejpam/article/view/1170

Burr, I. W. (1942). Cumulative frequency functions. Annals of Mathematical Statistics, 13(2), 215-232. doi: 10.1214/aoms/1177731607

El-Saidi, M. A., \& George, E. O. (1990). A generalized logistic model for quantal response. Biometrical Journal, 32(8), 943-954. doi:

10.1002/bimj.4710320807

Garthwait, P. H., Jolliffe, I. P., \& Jones, B. (1995). Statistical inference. London: Prentice Hall International.

Meeker, W. Q., \& Escobar, L. A. (1998). Statistical methods for reliability data. New York: John Wiley.

Mielke, W., Jr., \& Johnson, E. S. (1974). Some generalized beta distributions of the second kind having desirable application features in hydrology and meteorology. Water Resources Research, 10(2), 223-226. doi: 10.1029/WR010i002p00223

Pearson, K. (1895). X. Contributions to the mathematical theory of evolution.-II. Skew variations in homogeneous material. Philosophical Transactions of The Royal Society A, 186, 343-414. doi: 10.1098/rsta.1895.0010

Singh, S. K., \& Maddala, G. S. (1976). A function for the size distribution of incomes. Econometrica, 44(5), 963-970. doi: 10.1007/978-0-387-72796-7_2

Tadikamalla, P. R. (1980). A look at the Burr and related distributions. International Statistical Review, 48(3), 337-344. doi: 10.2307/1402945 